\title{
WHICH WEBSITE HAS THE BETTER FANTASY FOOTBALL PROJECTIONS: NFL.com OR ESPN?
}

\author{
Brett M. Dineen ${ }^{\mathrm{a}}$, David W. Packer ${ }^{\mathrm{a}}$, Michael C. Hayes ${ }^{\mathrm{a}}$, Matthew E. Bell ${ }^{\mathrm{a}}$, Paul M. Sommers ${ }^{\mathrm{a}}$
}

The authors use simple bilinear regression to assess the accuracy of projections made for fantasy football enthusiasts at the start of the 2015, 2016, and 2017 National Football League (NFL) seasons by two popular sites: NFL.com and ESPN. Comparisons reveal that over the three seasons combined NFL.com did a marginally better job predicting the future performance of running backs and wide receivers, while ESPN did a much better job predicting the future performance of quarterbacks and tight ends. The mode of analysis presented in this paper can be used to measure the accuracy of any site for any positional group of players in any given NFL season.

Keywords: National Football League, Fantasy Football, ESPN, simple regression

Every fall, just before the start of another National Football League (NFL) season, roughly a quarter of the U.S. population, including millions of women, sign up to play fantasy football [1]. The U.S. Supreme Court ruling in May 2018 that struck down federal limits on sports betting is likely to encourage even more participation in fantasy football where fans draft and run their own virtual football team.

Among the many fantasy football websites that provide projections for every NFL player, two of the most popular offering free advice are NFL.com and ESPN. However, which one provides projections that are more accurate?

In this paper, we use simple bilinear regression to assess the accuracy of the projections from the two aforementioned websites for quarterbacks, running backs, wide receivers, and tight ends in each of the three NFL seasons between 2015 and 2017. This method of analysis can be used to assess the accuracy of projections for any positional group of football players in any given year. The mode of analysis presented here can therefore help fantasy participants select which fantasy football league portal will best help them win a fantasy football championship.

\section{The Data}

Actual fantasy football points for the years 2015, 2016, and 2017 are from [2]. NFL.com's projected pre-season fantasy football points and ESPN's projected pre-season point totals for the same three regular seasons are from [3] and [4], respectively.
A typical fantasy football team includes one quarterback, two running backs, two wide receivers, one tight end, a defense/special team unit, a kicker and a "flex" (who may be a running back, wide receiver or tight end). A typical fantasy football league has between ten and twelve fantasy teams. (Obviously, a player drafted by one team cannot play for another team.) As a result, the starting players in a league would include ten to twelve different quarterbacks, twenty to thirty different running backs and wide receivers, and so forth. For each of the two websites - NFL.com and ESPN - we focused our attention on the respective preseason top 20 quarterbacks, top 40 running backs, top 40 wide receivers, and top 25 tight ends. The sample size for each group of players was based on the number of players at each position who could appear in a given week's lineup. The number of tight ends (25) exceeds the number of quarterbacks (20) because the "flex" player selected could be a tight end (or running back or wide receiver, but not another quarterback). Moreover, among the top preseason picks at each website, we included only players who played in at least twelve of the sixteen regular season games. Obviously, the projected point total for a player with fewer than sixteen (let alone twelve) games (say, because of an injury during the season or suspension for egregious violations of the NFL's personal conduct policy) would likely exceed his actual end-of-season point total. Tom Brady's (New England Patriots) four-game suspension at the start of the 2016 season might explain why Brady does not appear among the top 20 quarterbacks on either website's pre-season projections.

Projected and actual points in fantasy football use a standard scoring formula defined as follows:

.1 (rushing yards) +.1 (receiving yards) +.04 (passing yards)

+6 (rushing touchdowns) +6 (receiving touchdowns) +4 (passing touchdowns)

- 2 (interceptions thrown) - 2 (fumbles lost)

For example, Russell Wilson, starting quarterback for the Seattle Seahawks, in 2015 (see http://www.nfl.com/player/russell_wilson/2532975/profile) had 553 rushing yards, 4024 passing yards, one rushing touchdown, no receiving touchdowns, 34 passing touchdowns, 8 interceptions thrown, and 3 fumbles lost for an actual fantasy football point total of $336.26(=.1 \times 553+.04 \times 4024+6 \times$ $1+4 \times 34-2 \times 8-2 \times 3)$. 
Table 1. Actual and Projected Points, 2015-2017, Summary Statistics

\begin{tabular}{|c|c|c|c|c|}
\hline & $\begin{array}{l}\text { Quarterbacks } \\
\quad(\mathrm{n}=52)\end{array}$ & $\begin{array}{c}\text { Running Backs } \\
\quad(\mathrm{n}=79)\end{array}$ & $\begin{array}{l}\text { Wide Receriers } \\
\quad(\mathrm{n}=94)\end{array}$ & $\begin{array}{c}\text { Tight Ends } \\
(\mathrm{n}=55)\end{array}$ \\
\hline \multicolumn{5}{|l|}{ Actual points } \\
\hline Mean & 261.38 & 151.01 & 136.03 & 92.22 \\
\hline Std. Dev. & 50.89 & 59.10 & 44.02 & 35.63 \\
\hline Minimum & 133.4 & 40.6 & 37.6 & 17 \\
\hline Maximum & 389.08 & 325.8 & 245.1 & 183.6 \\
\hline \multicolumn{5}{|l|}{ Projected points } \\
\hline Mean & 276.44 & 164.21 & 156.47 & 98.90 \\
\hline Std. Dev. & 29.63 & 42.73 & 31.80 & 25.48 \\
\hline Minimum & 216.64 & 82.1 & 109.7 & 65 \\
\hline Maximum & 367.84 & 274.9 & 246.8 & 173.3 \\
\hline
\end{tabular}

Website: ESPN

\begin{tabular}{|c|c|c|c|c|}
\hline & $\begin{array}{l}\text { Quarterbacks } \\
\quad(\mathrm{n}=51)\end{array}$ & $\begin{array}{l}\text { Running Backs } \\
\quad(\mathrm{n}=86)\end{array}$ & $\begin{array}{l}\text { Wide Receriers } \\
\quad(\mathrm{n}=100)\end{array}$ & $\begin{array}{c}\text { Tight Ends } \\
(\mathrm{n}=55)\end{array}$ \\
\hline \multicolumn{5}{|l|}{ Actual points } \\
\hline Mean & 259.93 & 151.08 & 134.59 & 91.99 \\
\hline Std. Dev. & 52.36 & 59.73 & 47.45 & 35.28 \\
\hline Minimum & 133.4 & 40.6 & 32.3 & 13.2 \\
\hline Maximum & 389.08 & 325.8 & 245.1 & 183.6 \\
\hline \multicolumn{5}{|l|}{ Projected points } \\
\hline Mean & 283.05 & 165.45 & 147.03 & 97.65 \\
\hline Std. Dev. & 22.85 & 37.87 & 27.04 & 22.13 \\
\hline Minimum & 240.42 & 103.9 & 112.8 & 68.71 \\
\hline Maximum & 340.74 & 287.87 & 248.5 & 170.42 \\
\hline
\end{tabular}

Table 1 summarizes for each of the groups of players quarterbacks, running backs, wide receivers, and tight ends included in each projection (either NFL.com or ESPN), the number of observations for each group for the three seasons (2015, 2016, and 2017) combined, the mean, standard deviation, minimum, and maximum number of actual and projected fantasy football points. For each group of players and for both websites, the average number of projected points exceeded the average number of actual points. Projections are typically based on the assumption that most players (save for the most injury-prone) will play a full 16-game regular season while our samples included players who played fewer than 16 games (but no fewer than 12). In fact, only 32 (31) quarterbacks, 36 (38) running backs, 53 (54) wide receivers, and 28 (25) tight ends played in all 16 games all three seasons in our comparisons involving NFL.com (ESPN). Not surprisingly, the standard deviation of the projected number of points for each group of players on either website was always smaller than the corresponding standard deviation of the actual number of points. (That is, the dispersion of points will be smaller if one assumes that all players appear in 16 games.) Moreover, note that the minimum number of actual points was invariably smaller than the corresponding minimum number of projected points. Again, this result might also be the consequence of including players in our sample who played in less than a full 16-game regular season. For example, the minimum number of actual points for wide receivers is 37.6 over the three-year period (Kenny Britt of the 2017 Cleveland Browns who played in only 12 games), although the corresponding NFL.com projected minimum is 109.7 (Larry Fitzgerald of the 2017 Arizona Cardinals who played in all 16 regular season games). 


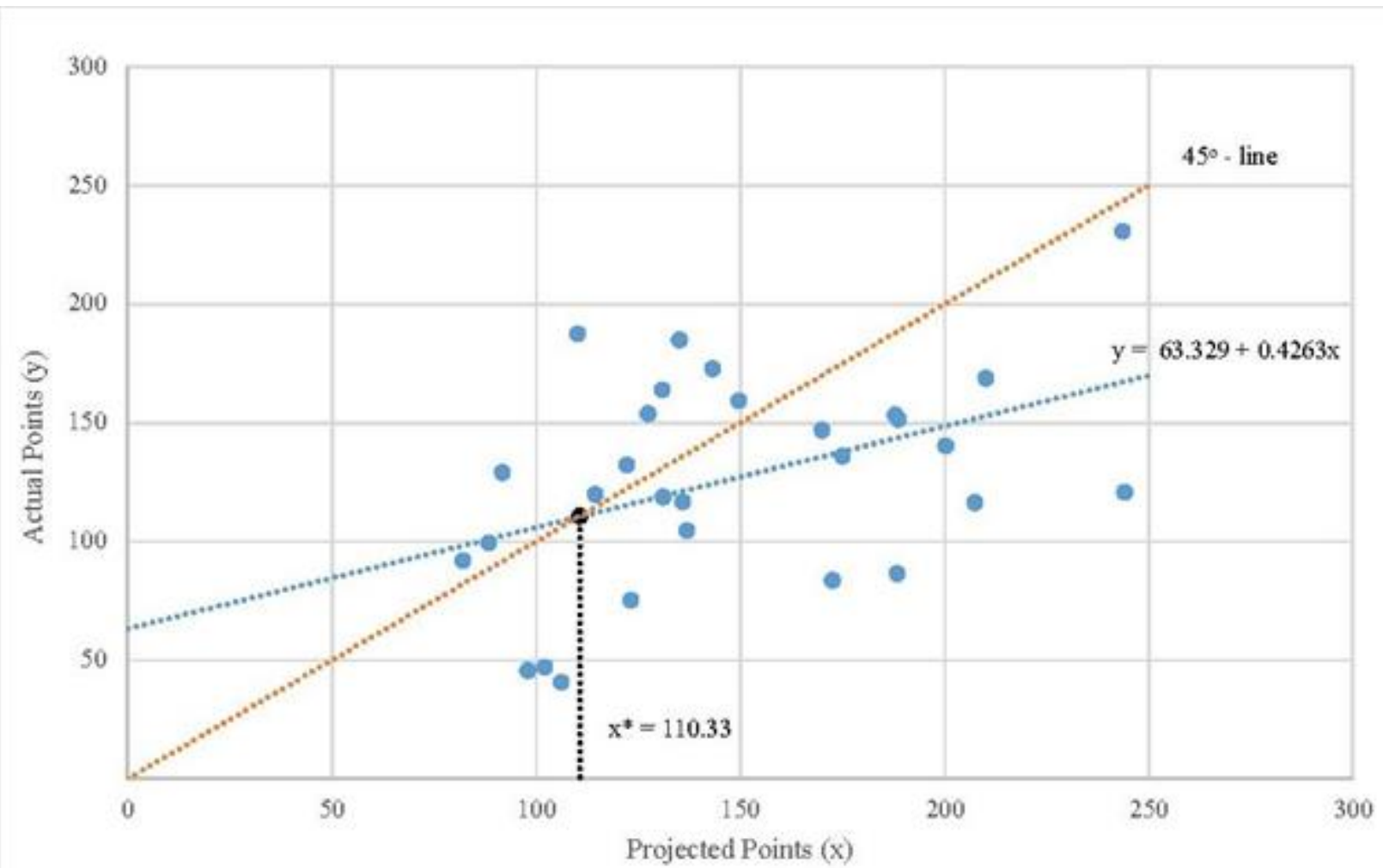

Figure 1. Actual Points vs. NFL.com Projected Points for Running Backs, 2015

Figure 1 shows a scatterplot of the actual number of fantasy football points for the highest rated running backs in the 2015 regular season versus the number of fantasy football points projected by NFL.com for these same players before the start of the 2015 season. Each point represents a running back whose actual points can be read along the vertical axis and whose projected point totals can be read along the horizontal axis. How strong is the relationship between actual and projected points? The estimated regression equation that summarizes this relationship would be given by:

$$
\text { Actual_FFP }=\mathrm{b}_{0}+\mathrm{b}_{1} \text { Projected_FFP }
$$

where $F F P$ denotes fantasy football points.

If, for any of the four positions - quarterbacks, running backs, wide receivers, or tight ends - the actual number of fantasy football points were equal to the projected number of fantasy football points, then all points in Figure 1 (where each point represents a running back) would fall on a 45-degree line (with a slope of 1.0), given by:

$$
\text { Actual_FFP }=\text { Projected_FFP }
$$

For equation (2), one can test the null hypothesis $\mathrm{H}_{0}$ : $\beta_{0}=$ 0 (against the two-tailed alternative, $\mathrm{H}_{\mathrm{A}}: \beta_{0} \neq 0$ ) using a $t$-test. The test statistic is $\mathrm{t}_{\text {Calc }}=b_{0} / S E\left(b_{0}\right)$, where $\mathrm{b}_{0}$ is the estimated constant term and $S E\left(b_{0}\right)$ is the standard error of the estimated constant term. Similarly, one can test the null hypothesis $\mathrm{H}_{0}$ : $\beta_{1}=1$ (against the two-tailed alternative,

$\left.\mathrm{H}_{\mathrm{A}}: \beta_{1} \neq 1\right)$. Here, the test statistic is tCalc $=\left(b_{1}-1\right) / S E\left(b_{1}\right)$, where $\mathrm{b}_{1}$ is the estimated slope coefficient and $S E\left(b_{1}\right)$ is the standard error of the estimated slope coefficient.

If we can reject $\mathrm{H}_{0}: \beta_{1}=1$ (using an $\alpha=.05$ level of significance), then the regression equation and the 45-degree line will intersect at a single point, where the actual number of fantasy football points is equal to the projected number of fantasy football points, hereafter called the critical point $\mathrm{x}^{*}$, where:

$$
\mathrm{x}^{*}=b_{0} /\left(1-b_{1}\right)
$$

If, as shown in Figure $1, x^{*}$ is equal to 110.33 , the regression analysis predicts that, on average, NFL.com overestimated the actual performance of running backs in 2015 with 110.33 or more actual fantasy football points. In fact, twenty of the 29 running backs in our sample that season accumulated more than 110.33 actual fantasy football points, a result that underscores the NFL.com's propensity to overestimate the performance of running backs that year.

If we cannot reject either $\mathrm{H}_{0}: \beta_{0}=0$ or $\mathrm{H}_{0}: \beta_{1}=1$ (in favor of their respective two-tailed alternative), then we would conclude that the regression line follows a 45-degree line, that is, projected points are roughly equal to actual points. 
Ultimately, the "more accurate" website will depend on the regression equation's coefficient of determination, $\mathrm{R}^{2}$ (the square of the simple correlation between actual and projected points); the higher the $\mathrm{R}^{2}$, the more accurate the website (that is, the usefulness of the website's regression equation for prediction of actual points depends on the strength of the association, namely, $\mathrm{R}^{2}$ ). If, for a given group of players, the $\mathrm{R}^{2}$ using one website's projected points is less than 10 percent higher than the $\mathrm{R}^{2}$ using the other website's projected points, we will conclude that the websites are the same. If one website yields an $\mathrm{R}^{2}$ that is 10 to 25 percent higher than the competing website, we will conclude that the website with the higher $\mathrm{R}^{2}$ is better. And, if the $\mathrm{R}^{2}$ is more than 25 percent higher than what we obtain using the other website, we will conclude that the former website's projections are much better.

Table 2. The Regression Results, NFL.com

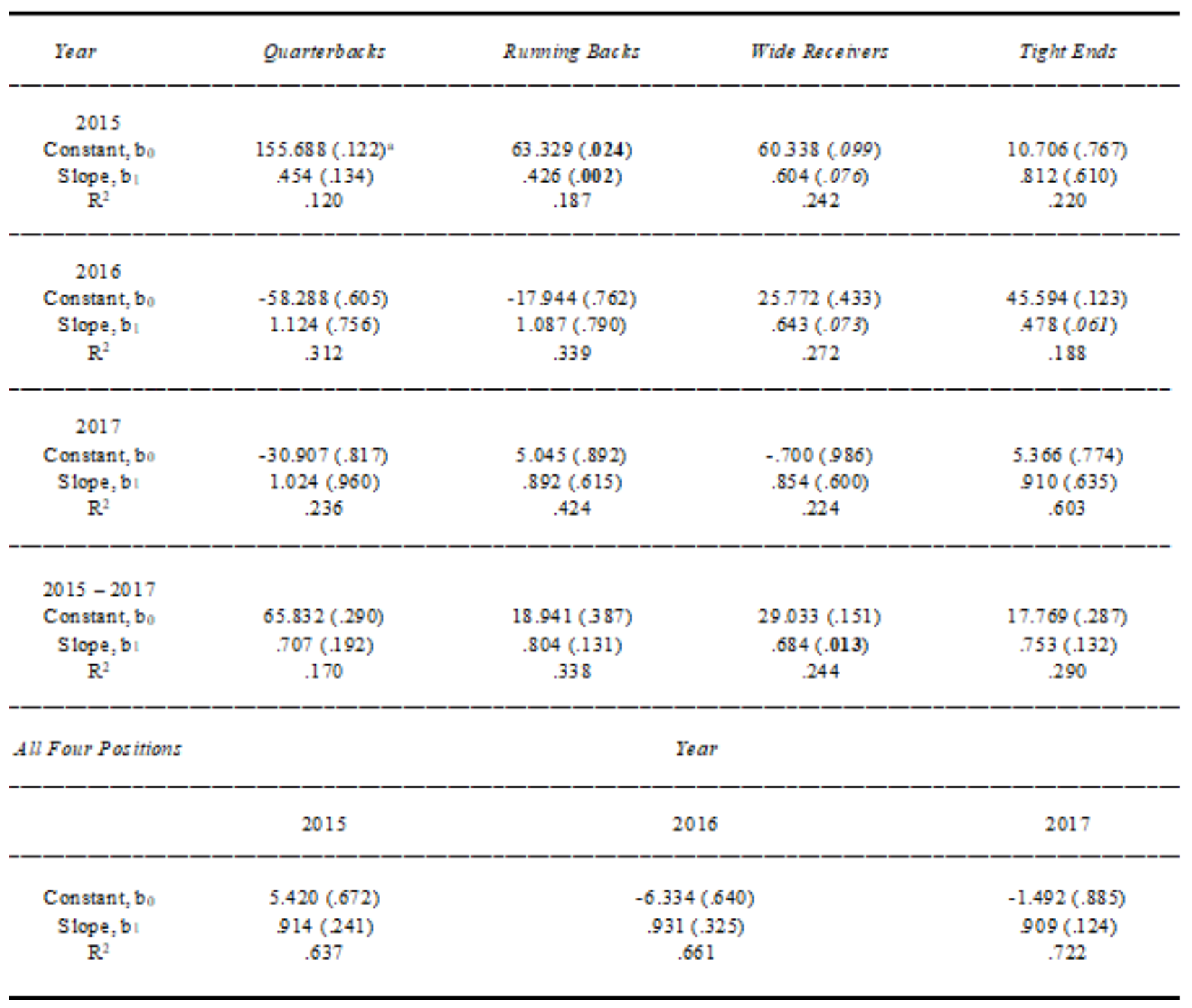

aThe numbers in parentheses are $p$-values. For the constant term, the $p$-value is for the null hypothesis that $\beta_{0}=0$ (against the two-tailed alternative that $\beta_{0} \neq 0$ ); for the slope coefficient, the $p$-value is for the null hypothesis that $\beta_{1}=1$ (against the two-tailed alternative that $\beta_{1} \neq 1$ ). 
Table 3. The Regression Results, ESPN

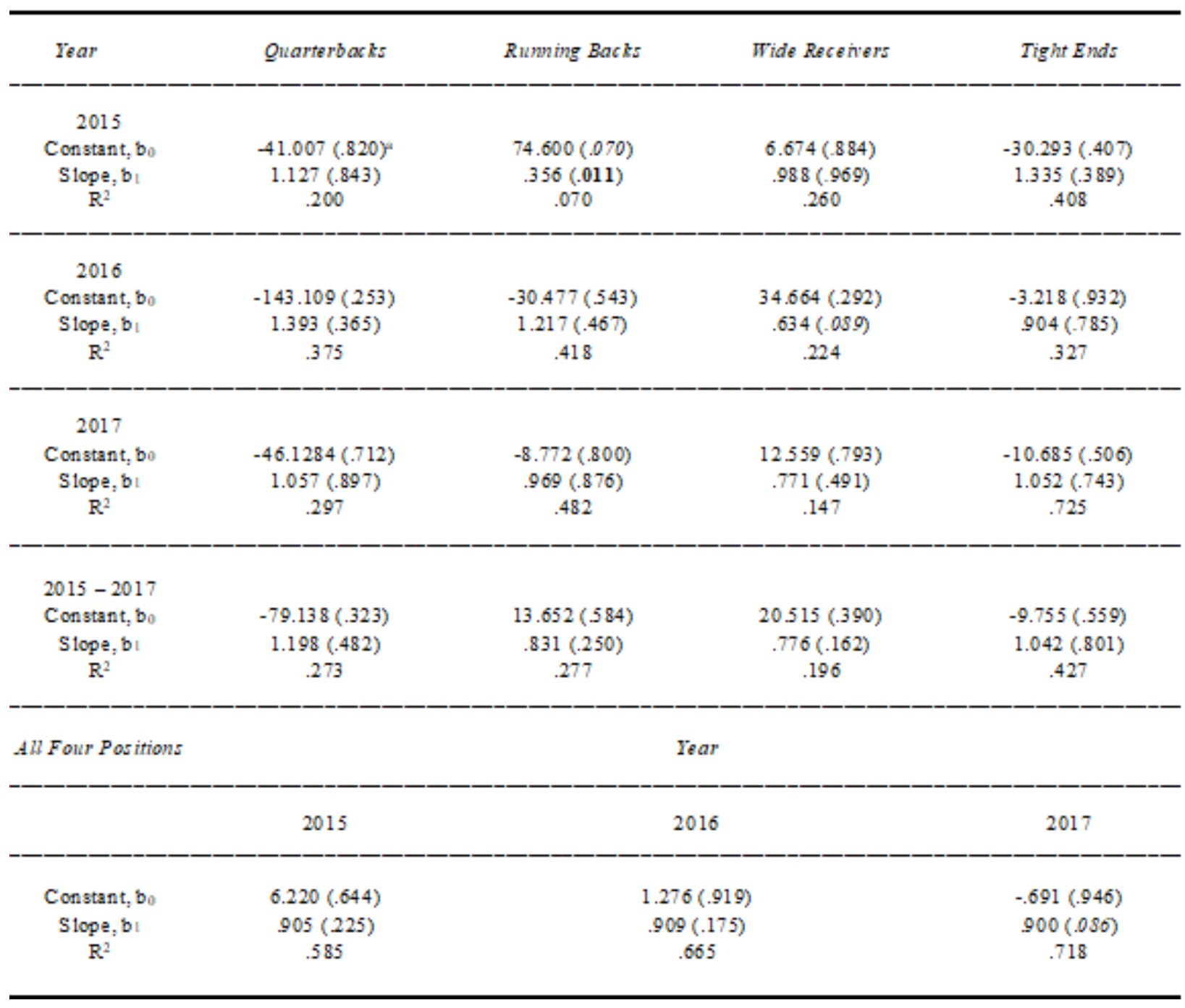

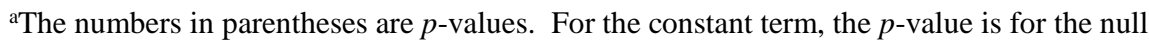
hypothesis that $\beta_{0}=0$ (against the two-tailed alternative that $\beta_{0} \neq 0$ ); for the slope coefficient, the $p$-value is for the null hypothesis that $\beta_{1}=1$ (against the two-tailed alternative that $\beta_{1} \neq 1$ ).

Tables 2 and 3 summarize the regression results relating actual points (the dependent variable) to projected points (the independent variable) for each of the four positions quarterbacks, running backs, wide receivers, and tight ends in each of the three seasons (2015 through 2017). Table 2 (based on projections at NFL.com) shows that in all but one of the twelve regressions reported in the first three rows of the table, the estimated regression line follows a 45-degree line passing through the origin. That is, the regression line has a constant term that is not discernibly different from zero and a slope coefficient that is not discernibly different from 1.0. The lone exception is the regression relating actual to projected points for running backs in 2015. When the observations on a single position are aggregated across all three seasons, the slope of the regression line for wide receivers is discernibly different from 1.0 (using an $\alpha=.05$ level of significance). In this instance, NFL.com appears to overestimate the performance of wide receivers whose actual point total exceeded $x^{*}=91.88$ points $[=29.033 /(1-.684)](77$ of the 94 wide receivers in our three-year sample). The coefficient of determination, $\mathrm{R}^{2}$, was highest for tight ends in 2017 and lowest for quarterbacks in 2015.

Table 3 (based on projections from ESPN) shows actual points closely follow projected points in all instances except (as was the case with NFL.com) running backs in 2015. The critical point, $\mathrm{x}^{*}$ (beyond which projected exceeded actual) applied to running backs with more than 115.84 points [= $74.6 /(1-.356)]$ (23 of 27 running backs in the year 2015). The $\mathrm{R}^{2}$ was highest for tight ends in 2017 and lowest for running backs in 2015. Figure 2 shows how well ESPN projected points to approximate actual points for running backs in 2017. 


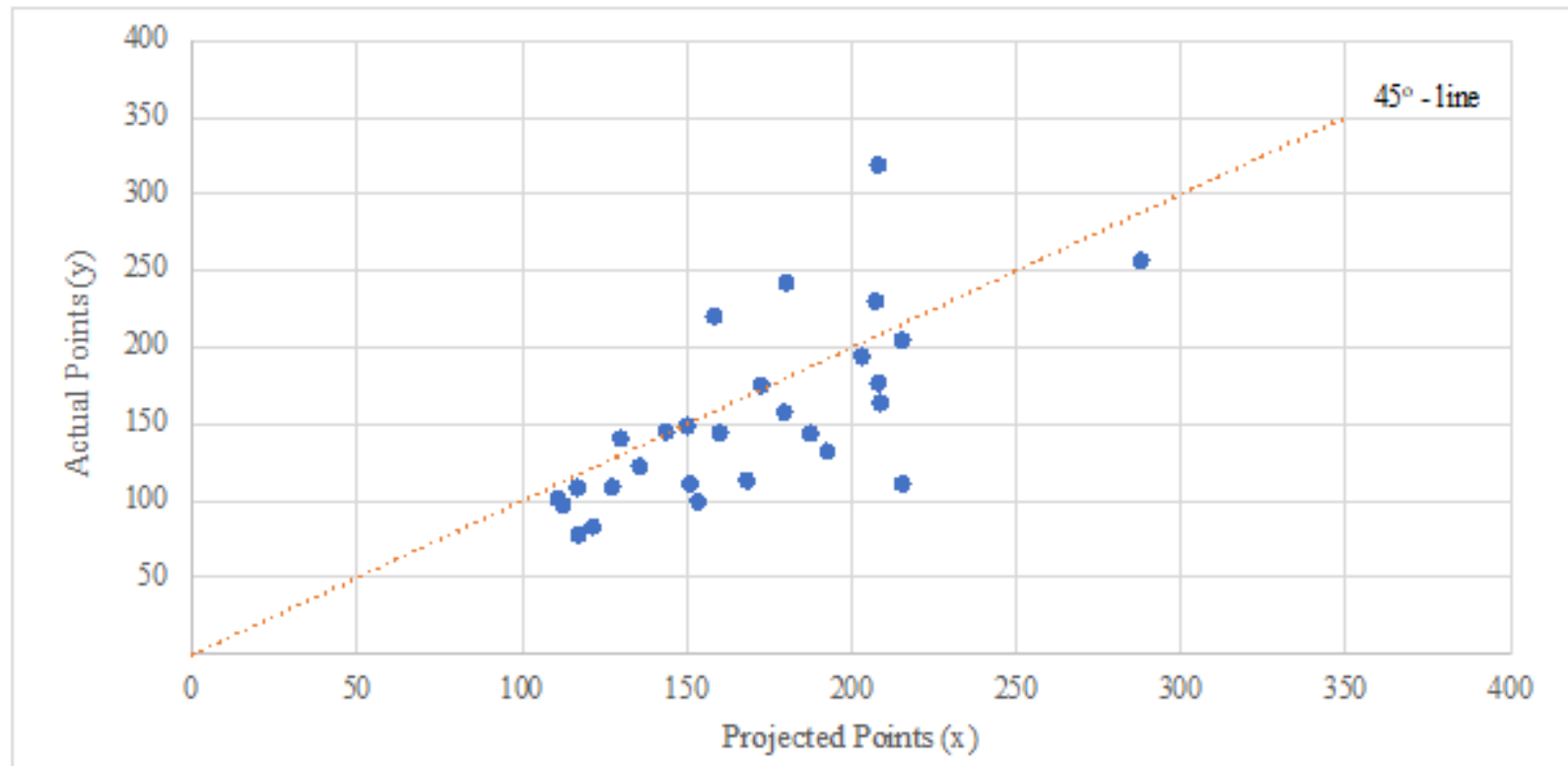

Figure 2. Actual Points vs. ESPN Projected Points for Running Backs, 2017

Table 4. Which Is The More Accurate Website, NFL.com Or ESPN?

\begin{tabular}{|c|c|c|c|c|}
\hline Year & Quarterbacks & Running Backs & Wide Recenvers & Tight Ends \\
\hline 2015 & $\mathrm{ESPN}^{a}$ & NFL.com & Same & ESPN \\
\hline 2016 & $E S P N$ & $E S P N$ & NFL com & ESPN \\
\hline 2017 & ESPN & $E S P N$ & NFL.com & $E S P N$ \\
\hline $2015-2017$ & ESPN & NFL com & NFL com & ESPN \\
\hline \multirow[t]{3}{*}{ All Four Pos itions } & \multicolumn{4}{|c|}{ Year } \\
\hline & 2015 & & 2016 & 2017 \\
\hline & Sane & & Same & Sane \\
\hline
\end{tabular}

af the difference in the $\mathrm{R}^{2}$ of the regressions related to that position between ESPN and NFL.com is less than 10 percent, the two websites are rated the Same; if the difference in the $\mathrm{R}^{2}$ is $10-25$ percent, the name of the more accurate website is in italics; and if the difference in the $\mathrm{R}^{2}$ is more than 25 percent, the name of the more accurate website is in boldface. 
Table 4 attempts to assess the accuracy of the two websites through a comparison involving the $\mathrm{R}^{2}$ of each regression at each position in each of the three seasons. The fourth row of results in Table 4 corresponding to three-year summaries suggests that for each of the four positions, ESPN has done the better job predicting how well quarterbacks and tight ends will perform, while NFL.com performed marginally better predicting the future performance of running backs and wide receivers. The last line in Table 4 corresponding to annual summaries across all four positions is a wash. That is, neither website gives the fantasy football player a clear advantage. Moreover, these annual summaries might be of little interest to fantasy football participants who have to select one or more players at each position. In 2017, the most recent year we examined, NFL.com did the better job of identifying wide receivers; ESPN did the better job of identifying quarterbacks, running backs, and tight ends.

\section{Concluding Remarks}

One can gauge the accuracy of fantasy football projections across different websites. This can be done by means of simple bilinear regressions between a player's actual number of fantasy football points (using a comprehensive measure of the player's performance based on rushing/receiving and passing yards, rushing/receiving and passing touchdowns, and turnovers) and a website's projected number of fantasy football points.

The two websites examined here are NFL.com and the exclusively sports network ESPN. For the three NFL regular seasons between 2015 and 2017, both websites, on average, projected more points than actual points for quarterbacks, running backs, wide receivers, and tight ends.

Based on the strength of the association between actual and projected points (using a regression's $\mathrm{R}^{2}$ ), the results varied by position. ESPN more accurately predicted the future performance of quarterbacks and tight ends over the three-year period; NFL.com (but only marginally) more accurately predicted the future performance of running backs and wide receivers.

\section{References}

1. G. Bresiger, "Nearly $75 \mathrm{M}$ people will play fantasy football this year,"

September 5, 2015, New York Post. Retrieved from: https://nypost.com/2015/09/05/nearly-75m-peoplewill-play-fantasy-football-this-year/

2. Actual points, retrieved from: https://fantasydata.com/nfl-stats/nfl-fantasyfootball-

stats.asp $x$ ?fs $=0 \&$ stype $=0 \&$ sn $=0 \&$ scope $=0 \& w=0 \&$ e $\underline{\mathrm{w}}=0 \& \mathrm{~s}=\& \mathrm{t}=0 \& \mathrm{p}=2 \& \mathrm{st}=$ FantasyPoints $\& \mathrm{~d}=1 \& 1 \mathrm{~s}=\&$ $\underline{\text { live } \& \text { false } \& \text { pidfalse } \& \text { minsnaps }=4}$

3. NFL.com projected points:

2015 retrieved

from:http://www.nfl.com/fantasyfootball/story/0ap3 000000492132/article/2015-fantasy-footballprofiles-and-projections-qb-120

2016 retrieved

from:http://www.nfl.com/fantasyfootball/story/0ap3 000000675406/article/quarterback-fantasyprojections-for-all-32-teams

2017 retrieved

from:http://www.nfl.com/news/story/0ap300000082 1461/article/fabianos-2017-fantasy-football-

projections-qbs

4. $\quad$ ESPN projected points:

2015 retrieved

from:http://games.espn.com/ffl/tools/projections?lea gueId $=465664 \&$ season Totals $=$ true $\&$ season $I d=2015$

2016 retrieved

from:http://games.espn.com/ffl/tools/projections?lea gueId $=465664 \&$ seasonTotals $=$ true $\&$ season $I d=2016$

2017 retrieved

from:http://games.espn.com/ffl/tools/projections?lea gueId $=465664 \&$ seasonTotals $=$ true $\&$ season $I d=2017$ 